\title{
Corrigendum
}

\section{RATIONAL POINTS ON INTERSECTIONS OF CUBIC AND QUADRIC HYPERSURFACES - CORRIGENDUM}

(doi:10.1017/S1474748014000127, published online 5 June 2014)

T. D. BROWNING, R. DIETMANN AND D. R. HEATH-BROWN

Keywords: Hasse principle; cubic; quadratic; system; rational point; Diophantine equations; circle method; Weyl sum; van der Corput method; complete intersection; corrigendum

In the article by Browning et al. [1] Lemma 9.1 was given incorrectly.

It is reproduced correctly below:

Lemma 9.1. If $\operatorname{ord}_{Q}(C) \geqslant 10, h_{Q}(C) \geqslant 2$, and $\rho \geqslant 23$, then we have $X_{\mathrm{sm}}\left(\mathbb{Q}_{p}\right) \neq \emptyset$ for every prime $p$.

Located under the heading ' 10 . Proof of Lemmas 9.1-9.5' the sentence 'Consider the projection $X \rightarrow \mathbb{P}^{n-2}$ from the point $[1,0, \ldots, 0]$.' should have read:

'If $Q_{1}$ vanishes identically it suffices to take a smooth $p$-adic point on $C_{1}=0$ with $x_{2} \neq 0$. Otherwise we consider the projection $X \rightarrow \mathbb{P}^{n-2}$ from the point $[1,0, \ldots, 0]$.'

\section{Reference}

1. T. D. Browning, R. Dietmann and D. R. Heath-Brown, Rational points on intersections of cubic and quadric hypersurfaces, J. Inst. Math. Jussieu Published by Cambridge University Press 5 June 2014, doi:10.1017/S1474748014000127. 